\title{
Darwin and mass-velocity relativistic corrections in the non-Born-Oppenheimer calculations of pure vibrational states of $\mathrm{H}_{2}$
}

\author{
Dariusz Kȩdziera \\ Department of Chemistry, Nicholas Copernicus University, ul. Gagarina 7, PL 87-100 Toruń, Poland \\ and Department of Chemistry, University of Arizona, Tucson, Arizona 85721
}

Monika Stanke

Institute of Physics, Nicholas Copernicus University, ul. Grudziadzka 5, PL 87-100 Toruń, Poland

and Department of Chemistry, University of Arizona, Tucson, Arizona 85721

Sergiy Bubin ${ }^{\text {a) }}$

Department of Physics, University of Arizona, Tucson, Arizona 85721 and Department of Chemistry, University of Arizona, Tucson, Arizona 85721

\author{
Maria Barysz \\ Department of Chemistry, Nicholas Copernicus ul. Gagarina 7, PL 87-100 Toruń, Poland \\ Ludwik Adamowicz \\ Department of Chemistry, University of Arizona, Tucson, Arizona 85721 and Department of Physics, \\ University of Arizona, Tucson, Arizona 85721
}

(Received 6 March 2006; accepted 9 May 2006; published online 7 July 2006)

\begin{abstract}
The Darwin and mass-velocity relativistic corrections have been calculated for all pure vibrational states of the $\mathrm{H}_{2}$ using the perturbation theory and very accurate variational wave functions obtained without assuming the Born-Oppenheimer (BO) approximation. Expansions in terms of explicitly correlated Gaussians with premultipliers in the form of even powers of the internuclear distance were used for the wave functions. With the inclusion of the two relativistic corrections to the non-BO energies the transition energies for the highest states agree more with the experimental results. () 2006 American Institute of Physics. [DOI: 10.1063/1.2209691]
\end{abstract}

\section{INTRODUCTION}

In our previous work ${ }^{1}$ we used 3000 explicitly correlated Gaussian functions (ECGF) with premultipliers in the form of even powers of the internuclear distance and calculated all 15 vibrational states of the $\mathrm{H}_{2}$ molecule with the zero total angular momentum. The calculations did not assume the Born-Oppenheimer (BO) approximation regarding the separability of the electronic and nuclear motions. In that work we demonstrated that the ECGFs are very effective in describing the coupled motion of the electrons and nuclei in $\mathrm{H}_{2}$. With 3000 ECGFs per state the total energies were converged very closely to the best available results for $\mathrm{H}_{2}{ }^{2}$ that Wolniewicz obtained using a variation-perturbation method with basis functions that are more capable of describing the electron-nuclear cusp. In fact, for the ground and first excited states our energies were lower than those of Wolniewicz. However, some small differences for highly excited states still remained. For example, for transitions $12 \rightarrow 11,13$ $\rightarrow 12$, and $14 \rightarrow 13$ we obtained the following results: 1415.187, 1049.269, and $622.063 \mathrm{~cm}^{-1}$, while the Wolniewicz's results were 1415.163, 1049.250, and $622.098 \mathrm{~cm}^{-1}$, respectively. In this work we revisited the problem and recalculated the $\mathrm{H}_{2}$ vibrational transitions with the basis of 5000 ECGFs per state. As it will be demon-

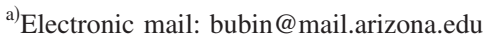

strated below, this considerably improved the agreement between our results and those of Wolniewicz. We also included the mass velocity and Darwin corrections in the calculations of the transition energies.

The most reliable experimental results concerning the pure vibrational transitions of $\mathrm{H}_{2}$ are still those of Dabrowski ${ }^{3}$ obtained more than two decades ago. While the accuracy of those results is probably not more than $0.1 \mathrm{~cm}^{-1}$ and the difference between the best calculated transition energies and the experimental transitions for the lower states oscilate both in terms of their magnitude and the sign, for the last four transitions the experimental energies are consistently lower than the calculated transitions (both ours and those of Wolniewicz). The only reason for this discrepancy can be the relativistic effects that were neglected in our non-BO calculations. Since we have recently developed procedures for calculating the mass-velocity and Darwin relativistic corrections to the non-BO energy, ${ }^{4,5}$ we decided to apply them to see if the calculations will yield results better agreeing with the experiment and narrowing the remaining gap between the theoretical and experimental transitions, particularly for the highest excited vibrational states.

The non-BO, nonrelativistic calculations presented in this work were performed using the method we have been developing in the last few years. For a description of the method, we refer the reader to our recent reviews. ${ }^{6,7}$ In this 
work we will briefly describe the method and its application to calculate the vibrational states of $\mathrm{H}_{2}$. We will also briefly describe the procedures used for calculating the massvelocity and Darwin corrections. It should be noted that the term "vibrational states" can only approximately describe states with zero total angular momentum. This is because if the Born-Oppenheimer approximation is not assumed, the vibrational motion is coupled with the electronic motion and, strictly speaking, the vibrational quantum number is not a good quantum number.

\section{THE NON-BO APPROACH}

The total nonrelativistic Hamiltonian for a system of $N$ particles (nuclei and electrons) has the following form in the laboratory Cartesian reference frame:

$$
\hat{H}_{\text {tot }}=-\sum_{i=1}^{N} \frac{1}{2 M_{i}} \nabla_{\mathbf{R}_{i}}^{2}+\sum_{i=1}^{N} \sum_{j>i}^{N} \frac{Q_{i} Q_{j}}{R_{i j}},
$$

where the masses, charges, and positions of the particles are denoted as $M_{i}, Q_{i}$, and $\mathbf{R}_{i}$, respectively. $\hat{H}_{\text {tot }}$ can be rigorously separated into the Hamiltonian describing the motion of the center-of-mass and an internal Hamiltonian. In our approach we transform the laboratory coordinate system into a coordinate system that contains three laboratory coordinates of the center-of-mass and $3 N-3$ internal Cartesian coordinates. The center of the internal system is placed at one of the particles (usually the heaviest one) called the reference particle and the internal coordinate axes are made parallel to the axes of the laboratory coordinate frame. The other $n$ $=N-1$ particles are referred to the reference particle using the position vectors $\mathbf{r}_{i}, i=1, \ldots, n$. The internal Hamiltonian, $\hat{H}$, is

$$
\hat{H}=-\frac{1}{2}\left(\sum_{i=1}^{n} \frac{1}{m_{i}} \nabla_{\mathbf{r}_{i}}^{2}+\sum_{i=1}^{n} \sum_{i \neq j}^{n} \frac{1}{M_{1}} \nabla_{\mathbf{r}_{i}}^{\prime} \nabla_{\mathbf{r}_{j}}\right)+V(\mathbf{r}),
$$

where

$$
V(\mathbf{r})=\sum_{i=1}^{n} \frac{q_{0} q_{i}}{r_{i}}+\sum_{j>i}^{n} \frac{q_{i} q_{j}}{r_{i j}},
$$

and where $q_{0}$ is the charge of the reference particle, $q_{i}(i$ $=1, \ldots, n)$ are the charges of the other $n$ particles, $r_{i j}=\mid \mathbf{r}_{j}$ $-\mathbf{r}_{i} \mid$, and, is used to indicate vector-matrix transposition. The separation of the internal Hamiltonian and the Hamiltonian of the motion of the center-of-mass is exact. The internal Hamiltonian (2) describes $n$ pseudoparticles with charges $q_{i}$ and reduced masses $m_{i}=M_{1} M_{i+1} /\left(M_{1}+M_{i+1}\right)$ moving in the spherically symmetric potential of charge $q_{0}$ of the reference particle. The motions of the pseudoparticles are coupled through the mass polarization term $\sum_{i \neq j}^{n} 1 / M_{1} \nabla_{\mathbf{r}_{i}}^{\prime} \nabla_{\mathbf{r}_{j}}$ and through the Coulombic interactions dependent on the distances of the pseudoparticles from the central charge, $r_{i}=\left|\mathbf{r}_{i}\right|$, and their relative distances, $r_{i j}$. In this work we number particles in such a way that particles 1 and 2 are nuclei, and particles 3 and 4 are electrons. Thus, in this scheme $r_{1}$ denotes the internuclear distance.
In our works concerning non-BO calculations on light diatomic molecular systems, ${ }^{1,8-17}$ we have shown that the explicitly correlated Gaussians (ECGs) involving functions with preexponential multipliers consisting of the internuclear distance, $\mathbf{r}_{1}$, raised to a non-negative even power, $m_{k}$

$$
\phi_{k}=r_{1}^{m_{k}} \exp \left[-\mathbf{r}^{\prime}\left(\mathbf{A}_{k} \otimes \mathbf{I}_{3}\right) \mathbf{r}\right]=r_{1}^{m_{k}} \exp \left[-\mathbf{r}^{\prime}\left(\overline{\mathbf{A}}_{k}\right) \mathbf{r}\right],
$$

where symbol $\overline{\mathbf{A}}$ denotes Kronecker product, $\overline{\mathbf{A}}=\mathbf{A} \otimes \mathbf{I}_{3}$, and $\mathbf{I}_{3}$ is the $3 \times 3$ identity matrix, very effectively describe nonadiabatic zero-angular-momentum states of those systems. Functions (4) are one-center correlated Gaussians with exponential coefficients forming the symmetric matrix $\mathbf{A}_{k}$ (unique for each basis function), which must be positive definite. The spatial part of the nonrelativistic wave function for each vibrational state is symmetric both with respect to interchange of the two protons (particles 1 and 2) and of the two electrons (particles 3 and 4). The permutational symmetry of the wave function is implemented by means of operators $\hat{P}_{i j}$. Their action on the Gaussians transforms the exponential parameters in the following way:

$$
\hat{P}_{i j} \phi_{k}=r_{1}^{m_{k}} \exp \left[-\mathbf{r}^{\prime}\left(\mathbf{T}_{P_{i j}}^{\prime} \mathbf{A}_{k} \mathbf{T}_{P_{i j}} \otimes I_{3}\right) \mathbf{r}\right],
$$

where $T_{P_{i j}}$ are the permutation matrices transforming the internal coordinates

$$
\begin{aligned}
& \mathbf{T}_{1}=\left(\begin{array}{lll}
1 & 0 & 0 \\
0 & 1 & 0 \\
0 & 0 & 1
\end{array}\right), \quad \mathbf{T}_{P_{12}}=\left(\begin{array}{lll}
-1 & 0 & 0 \\
-1 & 1 & 0 \\
-1 & 0 & 1
\end{array}\right), \\
& \mathbf{T}_{P_{34}}=\left(\begin{array}{lll}
1 & 0 & 0 \\
0 & 0 & 1 \\
0 & 1 & 0
\end{array}\right) .
\end{aligned}
$$

Properly symmetrized basis functions for $\mathrm{H}_{2}$ molecule are formed by applying the operator $\left(1+\hat{P}_{12}\right)\left(1+\hat{P}_{34}\right)$ to functions (4).

In the non-BO calculations we use the variational method. The energy and the wave function for each state are obtained by minimizing the Rayleigh quotient

$$
E\left(\left\{c_{k}\right\},\left\{m_{k}\right\},\left\{\mathbf{A}_{k}\right\}\right)=\min \frac{c^{\prime} H\left(\left\{m_{k}\right\},\left\{\mathbf{A}_{k}\right\}\right) c}{c^{\prime} S\left(\left\{m_{k}\right\},\left\{\mathbf{A}_{k}\right\}\right) c},
$$

with respect to the linear expansion coefficients of the wave function in terms of the basis functions, $c_{k}$, the basis function exponential parameters, $\left\{\mathbf{A}_{k}\right\}$, and the preexponential powers, $\left\{m_{k}\right\}$. The optimization is done separately for each state using an algorithm based on analytical derivatives of the energy, $E\left(\left\{c_{k}\right\},\left\{m_{k}\right\},\left\{\mathbf{A}_{k}\right\}\right)$, with respect to parameters $\mathbf{A}_{k}$. As mentioned before, in this work we used 5000 basis functions for each state. This is 2000 more than used in our previous non-BO calculations on $\mathrm{H}_{2}{ }^{1}$ We believe that with this many functions in the basis the ground state energy is converged to about 11 decimal figures, while the accuracy of the energy obtained for highly excited states is about 8 to 9 significant figures. This difference is due to increasingly more complicated structure of the "vibrational" part of the wave function for higher excited states, whose description requires a larger 
number of basis functions. The range of the preexponential powers, $\left\{m_{k}\right\}$, used was $0-250$, and these powers were partially optimized for each state. More details on the Hamiltonian transformation, proper symmetrization of the basis functions, and selection of nonlinear parameters for calculations with ECGFs can be found in Refs. 6 and 7.

\section{MASS-VELOCITY AND DARWIN RELATIVISTIC CORRECTIONS}

The increasing precision of the high resolution spectroscopy presents a challenge to the quantum mechanical calculations of molecular systems. The accuracy of the determination of the energies and the wave functions of the stationary states of those systems has to constantly increase to match the accuracy of the state-of-the-art experiments. At present it is not enough to only calculate the electronic component of the wave function with a very high precision, but it is also necessary to very accurately describe the motion of the nuclei (vibrational and rotational) and the coupling of the electronic and the nuclear motions. Furthermore, even for small systems, the relativistic effects have to be taken into account.

The simplest way to account for the relativistic corrections in atomic and molecular quantum systems is based on the Dirac-Coulomb-Breit-Pauli (DCBP) approximation. ${ }^{18}$ The DCBP approach provides a framework for describing a system of fermions (with spin 1/2) with the accuracy of the order $Z^{4} \alpha^{2}$, where $Z$ is the particle charge and $\alpha \approx 1 / 137$ is the fine structure constant. In this approximation the relativistic effects are treated as perturbations and determined as the first-order corrections to the nonrelativistic energy of the system using the nonrelativistic wave function as the zeroorder approximation. In our approach the nonrelativistic wave function depends on the internal coordinates of both the electrons and the nuclei forming the system since these two types of particles are treated on equal footing. Since we do not have any external potential, the mass-velocity correction and the spin-own-orbit interaction term are the only oneparticle relativistic corrections in the DCBP Hamiltonian. Among the two-particle corrections the most important is the Darwin term. The contributions due to the orbit-orbit and spin-other-orbit interactions are known to be much smaller ${ }^{19}$ and they can be neglected in the first approximation. Let us note, that the standard DCBP formulation is only valid for systems with spin $1 / 2$ particles. If the spin of a nuclei is different from $1 / 2$ then an extension of the basic formulation is necessary.

In the present work we consider the $\mathrm{H}_{2}$ molecule in its ground electronic (singlet) state. It is composed of two electrons and two protons. The standard DCBP formulation is valid in this case since all particles have spin of $1 / 2$. Moreover, since the total nuclear and electronic spins are equal to zero, the spin-orbit contribution vanishes. Also, since in $\mathrm{H}_{2}$ all the particles have unit charges, the largest relativistic corrections beyond the DCBP approximation is proportional to $\alpha^{4}$ and it is much smaller than the DCBP terms. Let us also note that the corrections derived from the quantum electrodynamics (QED) are of the order $\alpha^{3}$ and, thus, there are also relatively small. The relativistic contributions that we consid- ered in this work are the Darwin (D) and mass-velocity (MV) corrections. The largest neglected term is, therefore, the orbit-orbit interaction.

In this work the D and MV corrections have been calculated using the first-order perturbation theory and the Pauli approximation. In the calculations we used the nonrelativistic all-particle wave functions expressed in terms of explicitly correlated Gaussian functions obtained without assuming the BO approximation as described in the previous section. Our study differs from the previous studies where the relativistic corrections to the vibrational energies have been calculated by assuming the $\mathrm{BO}$ approximation and by averaging the electronic relativistic corrections over the vibrational wave functions. In our approach we have calculated these corrections not as purely electronic quantities, but as quantities due to both electrons and nuclei.

The Hamiltonians for the D and MV corrections in the laboratory coordinate frame are given by the following expressions:

$$
\begin{aligned}
& \hat{H}_{\mathrm{D}}=\frac{\alpha^{2}}{8} \sum_{i=1}^{N} \frac{1}{M_{i}^{2}} \sum_{j \neq i}^{N} \nabla_{\mathbf{R}_{i}}^{2} \frac{Q_{i} Q_{j}}{R_{i j}}, \\
& \hat{H}_{\mathrm{MV}}=-\frac{\alpha^{2}}{8} \sum_{i=1}^{N} \frac{1}{M_{i}^{3}} \nabla_{\mathbf{R}_{i}}^{4} .
\end{aligned}
$$

Upon the transformation of the laboratory coordinate system to the internal coordinate system, the Darwin Hamiltonian (8) separates into a term dependent on the position vector of the center-of-mass in the laboratory frame, $\mathbf{r}_{0}$, and a term dependent on the internal coordinates, $\{\mathbf{r}\}=\left\{\mathbf{r}_{1}^{\prime}, \mathbf{r}_{2}^{\prime}, \ldots, \mathbf{r}_{n}^{\prime}\right\}^{\prime}$

$$
\hat{H}_{\mathrm{D}}\left(\mathbf{r}, \mathbf{r}_{0}\right)=\hat{H}_{\mathrm{D}}\left(\mathbf{r}_{0}\right)+\hat{H}_{\mathrm{D}}(\mathbf{r}),
$$

where

$$
\hat{H}_{\mathrm{D}}\left(\mathbf{r}_{0}\right)=\frac{\alpha^{2}}{4} \frac{1}{m_{0}} \nabla_{\mathbf{r}_{0}}^{2} V(\mathbf{r})=0,
$$

because $V(\mathbf{r})$ is independent of $\mathbf{r}_{0}$, and where

$$
\begin{aligned}
\hat{H}_{\mathrm{D}}(\mathbf{r})= & \frac{\alpha^{2}}{8}\left[\sum_{i=1}^{n}\left(\frac{1}{M_{1}^{2}}+\frac{1}{M_{i+1}^{2}}\right) \nabla_{\mathbf{r}_{i}}^{2} \frac{q_{0} q_{i}}{r_{i}}\right. \\
& \left.+\sum_{i=1}^{n} \sum_{j \neq i}^{n} \frac{1}{M_{i+1}^{2}} \nabla_{\mathbf{r}_{i}}^{2} \frac{q_{i} q_{j}}{r_{i j}}\right] .
\end{aligned}
$$

Upon the same transformation of the coordinate system, $\{\mathbf{R}\} \rightarrow\left\{\mathbf{r}_{0}^{\prime}, \mathbf{r}^{\prime}\right\}^{\prime}$, the mass-velocity Hamiltonian can be represented as a sum of three terms

$$
\hat{H}_{\mathrm{MV}}\left(\mathbf{r}, \mathbf{r}_{0}\right)=\hat{H}_{\mathrm{MV}}\left(\mathbf{r}_{0}\right)+\hat{H}_{\mathrm{MV}}(\mathbf{r})+\hat{H}_{\mathrm{MV}}^{\mathrm{coupl}}\left(\mathbf{r}_{0}, \mathbf{r}\right),
$$

where the term $\hat{H}_{\mathrm{MV}}(\mathbf{r})$, relevant to the present calculations of the relativistic contributions, has the form

$$
\hat{H}_{\mathrm{MV}}(\mathbf{r})=-\frac{\alpha^{2}}{8}\left[\frac{1}{M_{1}^{3}}\left(\sum_{i=1}^{n} \nabla_{\mathbf{r}_{i}}\right)^{4}+\sum_{i=1}^{n} \frac{1}{M_{i+1}^{3}} \nabla_{\mathbf{r}_{i}}^{4}\right] .
$$

The last term in Eq. (13), $\hat{H}_{\mathrm{MV}}^{\text {coupl }}\left(\mathbf{r}_{0}, \mathbf{r}\right)$, describes relativistic coupling between the motion of the center-of-mass and the 
TABLE I. Nonrelativistic, non-BO transition energies computed in this work, in comparison with the experimental frequencies of Dabrowski [3] and with the nonrelativistic frequencies of Wolniewicz. (Ref. 12). All values are in $\mathrm{cm}^{-1}$. The values in parantheses are differences with respect to the data in the first column.

\begin{tabular}{cccc}
\hline \hline$v$ & $E_{v}^{\text {nonrel }}-E_{v+1}^{\text {nonrel }}$ & Experimental $($ Ref. 3) $(\Delta)$ & Wolniewicz $($ Ref. 2) $(\Delta)$ \\
\hline 0 & 4161.164 & $4161.14(0.02)$ & $4161.163(0.001)$ \\
1 & 3925.839 & $3925.79(0.05)$ & $3925.837(0.002)$ \\
2 & 3695.395 & $3695.43(-0.03)$ & $3695.392(0.003)$ \\
3 & 3467.986 & $3467.95(0.04)$ & $3467.983(0.003)$ \\
4 & 3241.581 & $3241.61(-0.03)$ & $3241.577(0.004)$ \\
5 & 3013.872 & $3013.86(0.01)$ & $3013.869(0.003)$ \\
6 & 2782.166 & $2782.13(0.04)$ & $2782.161(0.005)$ \\
7 & 2543.213 & $2543.25(-0.04)$ & $2543.209(0.004)$ \\
8 & 2292.997 & $2292.93(0.07)$ & $2292.993(0.004)$ \\
9 & 2026.408 & $2026.38(0.03)$ & $2026.406(0.002)$ \\
10 & 1736.778 & $1736.66(0.12)$ & $1736.776(0.002)$ \\
11 & 1415.162 & $1415.07(0.09)$ & $1415.163(-0.001)$ \\
12 & 1049.247 & $1049.16(0.09)$ & $622.098(-0.010)$ \\
13 & 622.088 & $622.02(0.07)$ & \\
\hline \hline
\end{tabular}

internal motion. This effect was not considered in our calculations as we assume that the molecule as a whole is at rest.

The calculation of the relativistic corrections to the energy of the internal motion of the system is performed for each state using the first-order perturbation theory as the expectation value of the Hamiltonian representing the internal mass-velocity and Darwin contributions

$$
\hat{H}^{\prime}(\mathbf{r})=\hat{H}_{\mathrm{MV}}(\mathbf{r})+\hat{H}_{\mathrm{D}}(\mathbf{r}) .
$$

After the wave functions for all $15(v=0, \ldots, 14)$ states of $\mathrm{H}_{2}$ were generated, we calculated the expectation value of the relativistic Hamiltonian (13) for each state and added it to the variational energy of that state. These energies, that include relativistic corrections, were used to calculate the transition energies. In the calculations we used the ratio of the proton-electron mass $m_{p} / m_{e}=1836.15267261$. The fine structure constant was $\alpha=1 / 137.03599911$. These values were taken from Ref. 20.

\section{RESULTS}

The first set of results is shown in Table I. It includes the data for the vibrational transition energies obtained in this work, as well as the experimental frequencies of Dabrowski, ${ }^{3}$ and nonrelativistic frequencies computed before by Wolniewicz. ${ }^{2}$ The values in parentheses show the difference between our results and the results obtained in the experiment and the calculated results of Wolniewicz, respectively. The latter difference indicates that our calculations produced essentially identical transition frequencies as those obtained by Wolniewicz. Some small positive differences that appear for the transitions up to $v=10$ (none of them larger than $0.005 \mathrm{~cm}^{-1}$ ) indicate that our non-BO energies are likely to be slightly less tightly converged than the energies of Wolniewicz. As mentioned, it usually takes more basis functions to obtain the same quality of the results for higher excited states than for the lower states, because of the higher number of nodes in the wave functions of the higher states. In the case of slightly lower accuracy of the calculated energy value for the $v+1$ state than for the $v$ state, the transi- tion frequency is slightly overestimated. This is the effect to which we attribute the small differences between our and Wolniewicz's values. However, for the highest three transitions, our transition frequencies are lower than those of Wolniewicz and the difference increases to $-0.010 \mathrm{~cm}^{-1}$ for the highest transition. Whether this is due to the lower precision of Wolniewicz's results for the states at the top of the vibrational spectrum or due to possible artifacts in the nonlinear parameter optimization for highly excited states in our calculations (i.e., when for some reason the energy is better converged for the $v+1$ state than for the $v$ state) is not immediately clear.

The comparison of our non-BO transition energies with the experimental transitions of Dabrowski, presented in Table I, shows alternating signs of the difference between our results and those of Dabrowski for the transitions up to $v=8$ to 9 . These variations are consistent with the error bracket of the experimental values estimated for about $0.1 \mathrm{~cm}^{-1}$. However, for the four highest transitions $(v$ $=10, \ldots, 13)$, the calculated non-BO frequencies are consistently higher than the experimental transitions by about $0.1 \mathrm{~cm}^{-1}$. This indicated to us that the relativistic effects should be taken into account to reduce the discrepancy between the experimentally and theoretically predicted transitions. As described in the previous section, the relativistic corrections that we have calculated include the mass-velocity and Darwin effects. These are the major relativistic effects for states of diatomic molecules where the electronic density of the two atoms (in the present case hydrogen atoms) are not too distorted from the spherical symmetry due to the formation of a chemical bond. The near spherical symmetry of the electronic distribution around the two atoms reduces the relativistic effects dependent on the angular momentum (the spin-orbit and orbit-orbit interactions). For $\mathrm{H}_{2}$ the electronic distribution around the two atomic centers should be particularly symmetric in the highest vibrational states where the average distance between the nuclei is much larger than the equilibrium distance $\left(r_{e}\right.$, the distance that corresponds to the minimum of the potential energy curve in BO calcula- 
TABLE II. Non-BO, nonrelativistic (nonrel) and relativistically corrected (rel) vibrational frequencies $E_{v+1}-E_{v}$ for $v=12 \rightarrow 11, v=13 \rightarrow 12$, and $v$ $=14 \rightarrow 13$ transitions of $\mathrm{H}_{2}$ in comparison with the experimental transitions of Dabrowski (Ref. 3) and calculated frequencies obtained by Wolniewicz (Ref. 21 that include relativistic and radiative corrections. All values are in $\mathrm{cm}^{-1}$.)

\begin{tabular}{|c|c|c|c|c|}
\hline$v$ & $E_{v+1}^{\text {nonrel }}-E_{v}^{\text {nonrel }}$ & $E_{v+1}^{\mathrm{rel}}-E_{v}^{\mathrm{rel}}$ & $\begin{array}{l}\text { Previous } \\
\text { calculations } \\
\text { (Ref. 21) }\end{array}$ & $\begin{array}{c}\text { Experiment } \\
\text { (Ref. 3) }\end{array}$ \\
\hline 10 & 1736.778 & 1736.759 & 1736.707 & 1736.66 \\
\hline 11 & 1415.162 & 1415.128 & 1415.076 & 1415.07 \\
\hline 12 & 1049.247 & 1049.194 & 1049.139 & 1049.16 \\
\hline 13 & 622.088 & 622.015 & 621.956 & 622.02 \\
\hline
\end{tabular}

tions). Thus, for those highest vibrational states the account for the mass-velocity and Darwin effects is likely to improve the agreement between the calculated and experimental transition energies. This has been tested in the present calculations.

In Table II we show a comparison between the calculated transition frequencies obtained using the total energies of the vibrational states corrected and uncorrected for the relativistic effects with the experimental frequencies of Dabrowski. Only the top four transitions are shown in the table. Those are the transitions whose frequencies are likely to be overestimated by the non-BO calculations. After adding the relativistic corrections the agreement between the experimental results and the calculated values improves for all four transitions. The agreement is the best for the top $14 \rightarrow 13$ transition and, as expected, worsens as the vibrational quantum number decreases. For that top transition the non-BO frequency uncorrected for the relativistic effects is $622.088 \mathrm{~cm}^{-1}$ and the corrected one is $622.015 \mathrm{~cm}^{-1}$ which is very close to the experimental transition of $622.02 \mathrm{~cm}^{-1}$. In Table II we also included the transition frequencies obtained by Wolniewicz using his non-BO energies corrected for electronic relativistic and radiative effects calculated within the BO approximation. ${ }^{21}$ As one notices, while for the top two states our and Wolniewicz's results agree equally well with the experiment, for the next two states Wolniewicz's results are slightly better. We attribute this to the growing importance of the relativistic magnetic interactions which Wolniewicz included in his calculations and we have not. It should be remembered that the presented comparison is subject to the inaccuracy of the experimental transitions which was discussed before.

The relativistic corrections, the total non-BO energies uncorrected and corrected for the relativistic effects, as well as relativistically corrected transition frequencies for all 15 vibrational states of $\mathrm{H}_{2}$ obtained with 5000 ECGFs are shown in Table III. As one can notice, both mass-velocity and Darwin effects decrease with the vibrational excitation. For the highest state $(v=14)$ both corrections become very close to twice the respective values for the hydrogen atom as they should. Since imn this state the molecule is nearly dissociated it can be considered as a pair of weakly interacting, distant hydrogen atoms. For comparison purposes we also included in Table III the transition frequencies of Wolniewicz that include relativistic and radiative corrections. ${ }^{21}$

Besides comparing our transition energies with Wolniewicz's results that included all relativistic corrections he calculated, we also made a comparison of the electronelectron and electron-nucleus contributions to the Darwin correction with the values of those contributions obtained by Wolniewicz. This comparison was possible because we obtained from Wolniewicz his unpublished results concerning the contributions. ${ }^{2}$ There were two reasons why such a comparison was useful. First, it allowed us to verify the correctness of our results, and second, it provided a way of estimating the difference between those corrections calculated by averaging the electronic contributions over the vibrational wave functions (the approach used by Wolniewicz) versus calculating them directly from the non-BO wave functions (our approach). One can distinguish two types of the Darwin

TABLE III. Total non-BO energies, $E_{v}^{\text {nonrel }}$, mass-velocity, and Darwin relativistic corrections, total non-BO energy with the relativistic corrections included, $E_{v}^{\text {rel }}$ (all in a.u.), relativistically corrected transition frequencies computed in this work, $E_{v+1}^{\text {rel }}-E_{v}^{\text {rel }}$, and frequencies obtained by Wolniewicz (Ref. 21$)$ that include relativistic and radiative corrections (the latter two are in $\mathrm{cm}^{-1}$ ).

\begin{tabular}{|c|c|c|c|c|c|c|}
\hline$v$ & $E_{v}^{\text {nonrel }}$ & Mass-velocity & Darwin & $E_{v}^{\mathrm{rel}}$ & $E_{v+1}^{\mathrm{rel}}-E_{v}^{\mathrm{rel}}$ & Wolniewicz (Ref. 21) \\
\hline 0 & -1.16402503052 & $-8.657522 \times 10^{-5}$ & $7.281823 \times 10^{-5}$ & -1.16403878751 & 4161.238 & 4161.167 \\
\hline 1 & -1.14506537114 & $-8.414339 \times 10^{-5}$ & $7.072132 \times 10^{-5}$ & -1.14507879321 & 3925.904 & 3925.836 \\
\hline 2 & -1.12717793348 & $-8.188957 \times 10^{-5}$ & $6.876302 \times 10^{-5}$ & -1.12719106004 & 3695.452 & 3695.389 \\
\hline 3 & -1.11034047516 & $-7.980406 \times 10^{-5}$ & $6.693591 \times 10^{-5}$ & -1.11035334331 & 3468.035 & 3467.976 \\
\hline 4 & -1.09453916771 & $-7.787725 \times 10^{-5}$ & $6.523308 \times 10^{-5}$ & -1.09455181188 & 3241.622 & 3241.564 \\
\hline 5 & -1.07976943774 & $-7.609807 \times 10^{-5}$ & $6.364060 \times 10^{-5}$ & -1.07978189521 & 3013.905 & 3013.851 \\
\hline 6 & -1.06603722490 & $-7.447390 \times 10^{-5}$ & $6.216637 \times 10^{-5}$ & -1.06604953243 & 2782.190 & 2782.136 \\
\hline 7 & -1.05336074599 & $-7.298806 \times 10^{-5}$ & $6.079169 \times 10^{-5}$ & -1.05337294236 & 2543.228 & 2543.175 \\
\hline 8 & -1.04177301560 & $-7.164116 \times 10^{-5}$ & $5.951347 \times 10^{-5}$ & -1.04178514329 & 2293.002 & 2292.950 \\
\hline 9 & -1.03132535444 & $-7.043407 \times 10^{-5}$ & $5.832927 \times 10^{-5}$ & -1.03133745925 & 2026.402 & 2026.351 \\
\hline 10 & -1.02209235935 & $-6.936293 \times 10^{-5}$ & $5.723048 \times 10^{-5}$ & -1.02210449180 & 1736.759 & 1736.707 \\
\hline 11 & -1.01417901712 & $-6.843596 \times 10^{-5}$ & $5.621885 \times 10^{-5}$ & -1.01419123424 & 1415.128 & 1415.076 \\
\hline 12 & -1.00773106386 & $-6.766075 \times 10^{-5}$ & $5.528758 \times 10^{-5}$ & -1.00774343704 & 1049.194 & 1049.139 \\
\hline 13 & -1.00295034375 & $-6.704939 \times 10^{-5}$ & $5.443745 \times 10^{-5}$ & -1.00296295569 & 622.015 & 621.956 \\
\hline 14 & -1.00011590063 & $-6.659363 \times 10^{-5}$ & $5.364734 \times 10^{-5}$ & -1.00012884692 & & \\
\hline $\mathrm{H}+\mathrm{H}$ & -0.99945567942 & $-6.654970 \times 10^{-5}$ & $5.316445 \times 10^{-5}$ & -0.99946906467 & & \\
\hline
\end{tabular}


TABLE IV. Comparison of the Darwin electron-nucleus corrections, Darwin (e-n), and the Darwin electronelectron corrections, Darwin $(e-e)$, for pure vibrational states of $\mathrm{H}_{2}$ obtained in this work with 5000 explicitly correlated Gaussian functions for each state with the results obtained by Wolniewicz (Ref. 2). All results are in a.u.

\begin{tabular}{rcccc}
\hline \hline$v$ & Darwin $(e-n)$ & Wolniewicz (Ref. 2) & Darwin $(e-e)$ & Wolniewicz (Ref. 2) \\
\hline 0 & $7.552190 \times 10^{-5}$ & $7.561170 \times 10^{-5}$ & $-2.703663 \times 10^{-6}$ & $-2.707602 \times 10^{-6}$ \\
1 & $7.324601 \times 10^{-5}$ & $7.333604 \times 10^{-5}$ & $-2.524685 \times 10^{-6}$ & $-2.529222 \times 10^{-6}$ \\
2 & $7.111890 \times 10^{-5}$ & $7.120937 \times 10^{-5}$ & $-2.355883 \times 10^{-6}$ & $-2.358131 \times 10^{-6}$ \\
3 & $6.912886 \times 10^{-5}$ & $6.922121 \times 10^{-5}$ & $-2.192954 \times 10^{-6}$ & $-2.193420 \times 10^{-6}$ \\
4 & $6.726520 \times 10^{-5}$ & $6.736064 \times 10^{-5}$ & $-2.032115 \times 10^{-6}$ & $-2.033265 \times 10^{-6}$ \\
5 & $6.551729 \times 10^{-5}$ & $6.561897 \times 10^{-5}$ & $-1.876692 \times 10^{-6}$ & $-1.876299 \times 10^{-6}$ \\
6 & $6.388788 \times 10^{-5}$ & $6.398735 \times 10^{-5}$ & $-1.721504 \times 10^{-6}$ & $-1.720928 \times 10^{-6}$ \\
7 & $6.235619 \times 10^{-5}$ & $6.245893 \times 10^{-5}$ & $-1.564499 \times 10^{-6}$ & $-1.565329 \times 10^{-6}$ \\
8 & $6.092154 \times 10^{-5}$ & $6.102573 \times 10^{-5}$ & $-1.408074 \times 10^{-6}$ & $-1.407452 \times 10^{-6}$ \\
9 & $5.957435 \times 10^{-5}$ & $5.968070 \times 10^{-5}$ & $-1.245073 \times 10^{-6}$ & $-1.244791 \times 10^{-6}$ \\
10 & $5.830577 \times 10^{-5}$ & $5.841631 \times 10^{-5}$ & $-1.075287 \times 10^{-6}$ & $-1.074384 \times 10^{-6}$ \\
11 & $5.711155 \times 10^{-5}$ & $5.722415 \times 10^{-5}$ & $-8.927049 \times 10^{-7}$ & $-8.919026 \times 10^{-7}$ \\
12 & $5.598219 \times 10^{-5}$ & $5.609510 \times 10^{-5}$ & $-6.946106 \times 10^{-7}$ & $-6.923351 \times 10^{-7}$ \\
13 & $5.490705 \times 10^{-5}$ & $5.501502 \times 10^{-5}$ & $-4.695910 \times 10^{-7}$ & $-4.679356 \times 10^{-7}$ \\
14 & $5.385834 \times 10^{-5}$ & $5.396934 \times 10^{-5}$ & $-2.110071 \times 10^{-7}$ & $-2.091358 \times 10^{-7}$ \\
$\mathrm{H}+\mathrm{H}$ & $5.316445 \times 10^{-5}$ & & 0.0 & \\
\hline \hline
\end{tabular}

corrections, the electron-nucleus $(e-n)$ and the electronelectron $(e-e)$. In the $\mathrm{BO}$ calculation the former is calculated as a one-electron contribution and the latter as a two-electron contribution. However, in the non-BO approach (using the internal non-BO wave function that for $\mathrm{H}_{2}$ depends on the coordinates of three pseudoparticles) the former is a sum of two one-pseudoparticle contributions due to pseudoparticles 2 and 3 and two two-pseudoparticle contributions due to the 1-2 and 1-3 pseudoparticle pairs. The electron-electron contribution in the non-BO approach is calculated as a twopseudoparticle quantity due to the pseudoparticle pair 2-3. The comparison of the $e-n$ and $e-e$ Darwin corrections obtained in our calculations and those of Wolniewicz is presented in Table IV. As one notices the values are very close but not identical. In average they differ in the third significant figure. For the highest vibrational level $(v=14)$ the $e-n$ contribution becomes almost equal to twice the Darwin cor- rection for the hydrogen atom and the $e$-e contribution approaches zero. This is the trend one expects to see for those contributions.

Finally, in Table V we present the expectation values of the interparticle distances and their squares obtained with 5000 function basis sets. Those include proton-proton, proton-electron, and electron-electron distances. These results show that for the top states the internuclear and interelectronic distances are, as expected, considerably larger than for the bottom states and, thus, for those top states the magnetic relativistic corrections should be much smaller than the mass-velocity and Darwin corrections.

\section{SUMMARY}

In this work we used the recently developed algorithms for the mass-velocity and Darwin relativistic corrections to

TABLE V. Expectation values of the interparticle distances $\left(r_{1} \equiv r_{p p}, r_{2} \equiv r_{p e}, r_{23} \equiv r_{e e}\right)$ obtained with 5000 basis function sets. All values are in a.u.

\begin{tabular}{lcccccc}
\hline \hline$v$ & $\left\langle r_{1}\right\rangle$ & $\left\langle r_{2}\right\rangle$ & $\left\langle r_{23}\right\rangle$ & $\left\langle r_{1}^{2}\right\rangle$ & $\left\langle r_{2}^{2}\right\rangle$ & $\left\langle r_{23}^{2}\right\rangle$ \\
\hline 0 & 1.44874 & 1.57482 & 2.20133 & 2.12705 & 3.14539 & 5.80533 \\
1 & 1.54535 & 1.62571 & 2.26584 & 2.47400 & 3.36512 & 6.15743 \\
2 & 1.64606 & 1.67848 & 2.33448 & 2.85682 & 3.60174 & 6.54189 \\
3 & 1.75171 & 1.73354 & 2.40819 & 3.28141 & 3.85831 & 6.96610 \\
4 & 1.86342 & 1.79144 & 2.48828 & 3.75570 & 4.13894 & 7.44001 \\
5 & 1.98273 & 1.85290 & 2.57646 & 4.29054 & 4.44932 & 7.97723 \\
6 & 2.11176 & 1.91894 & 2.67508 & 4.90132 & 4.79754 & 8.59687 \\
7 & 2.25354 & 1.99104 & 2.78748 & 5.61053 & 5.19543 & 9.32646 \\
8 & 2.41259 & 2.07140 & 2.91847 & 6.45252 & 5.66100 & 10.20725 \\
9 & 2.59589 & 2.16340 & 3.07541 & 7.48237 & 6.22307 & 11.30392 \\
10 & 2.81493 & 2.27263 & 3.27007 & 8.79459 & 6.93083 & 12.72449 \\
11 & 3.09015 & 2.40903 & 3.52301 & 10.56675 & 7.87601 & 14.66611 \\
12 & 3.46263 & 2.59255 & 3.87464 & 13.18132 & 9.25452 & 17.54054 \\
13 & 4.03411 & 2.87265 & 4.42340 & 17.67911 & 11.59453 & 22.43467 \\
14 & 5.21022 & 3.44713 & 5.55825 & 28.91193 & 17.33540 & 34.27670 \\
\hline \hline
\end{tabular}


the non-Born-Oppenheimer energy of diatomic systems to calculate pure vibrational transitions of $\mathrm{H}_{2}$. In our approach these two relativistic effects are calculated in a framework where the electronic and nuclear motions in the molecule are not separated (as happens when the $\mathrm{BO}$ approximation is assumed). Thus the calculations describe on an equal footing the relativistic effects due to electrons and nuclei, as well as the relativistic effects due to interactions between these two types of particles.

The calculations of the vibrational transition energies focus on the highest transitions because, for those, the massvelocity and Darwin relativistic corrections are expected to be much more important than the relativistic corrections dependent on the angular momenta of the electrons. Also, for those states the differences between the non-BO transition energies calculated without the relativistic corrections and the experimental transition energies have the same sign and are of similar magnitude indicating that including the massvelocity and Darwin corrections should improve the agreement with the experiment and, as has been demonstrated by the present calculations, it did.

The work to follow will include the development of algorithms for calculating the magnetic spin-spin, spin-orbit, and orbit-orbit interactions. The orbit dependent relativistic corrections become more important for lower vibrational transitions of $\mathrm{H}_{2}$ where the atomic electronic distributions are more distorted from the spherical symmetry due to the formation of a covalent bond between the two hydrogen atoms.

\section{ACKNOWLEDGMENTS}

This work has been supported in part by the National Science Foundation. We would also like to thank the Univer- sity of Arizona Center of Computing and Information Technology for the computer time on the HP Alpha GS1280 parallel computer system.

${ }^{1}$ S. Bubin and L. Adamowicz, J. Chem. Phys. 118, 3079 (2003).

${ }^{2}$ L. Wolniewicz, (private communication); J. Chem. Phys. 99, 1851 (1993).

${ }^{3}$ I. Dabrowski, Can. J. Phys. 62, 1639 (1984).

${ }^{4}$ M. Stanke, D. Kędziera, M. Molski, S. Bubin, M. Barysz, and L. Adamowicz, Phys. Rev. Lett. 96, 233002 (2006).

${ }^{5}$ D. Kędziera, M. Stanke, S. Bubin, M. Barysz, and L. Adamowicz, J. Chem. Phys. (to be published).

${ }^{6}$ M. Cafiero, S. Bubin, and L. Adamowicz, Phys. Chem. Chem. Phys. 5, 1491 (2003).

${ }^{7}$ S. Bubin, M. Cafiero, and L. Adamowicz, Adv. Chem. Phys. 131, 377 (2005).

${ }^{8}$ D. B. Kinghorn and L. Adamowicz, J. Chem. Phys. 106, 4589 (1997).

${ }^{9}$ D. B. Kinghorn and L. Adamowicz, J. Chem. Phys. 110, 7166 (1999).

${ }^{10}$ D. B. Kinghorn and L. Adamowicz, Phys. Rev. Lett. 83, 2541 (1999).

${ }^{11}$ C. E. Scheu, D. B. Kinghorn, and L. Adamowicz, J. Phys. Chem. 114, 3393 (2001).

${ }^{12}$ S. Bubin and L. Adamowicz, J. Chem. Phys. 120, 6051 (2004).

${ }^{13}$ S. Bubin and L. Adamowicz, J. Chem. Phys. 121, 6249 (2004).

${ }^{14}$ S. Bubin, E. Bednarz, and L. Adamowicz, J. Chem. Phys. 122, 041102 (2005).

${ }^{15}$ E. Bednarz, S. Bubin, and L. Adamowicz, J. Chem. Phys. 122, 164302 (2005).

${ }^{16}$ M. Pavanello, S. Bubin, M. Molski, and L. Adamowicz, J. Chem. Phys. 123, 104306 (2005).

${ }^{17}$ S. Bubin, L. Adamowicz, and M. Molski, J. Chem. Phys. 123, 134310 (2005).

${ }^{18}$ H. A. Bethe and E. E. Salpeter, Quantum Mechanics of One- and TwoElectron Atoms (Plenum, New York, 1977).

${ }^{19}$ S. Fraga, J. Karwowski, and K. M. S. Saxena, Handbook of Atomic Data (Elsevier, Amsterdam, 1976).

${ }^{20}$ See CODATA 2002 recommended values at http://physics.nist.gov/cuu

${ }^{21}$ L. Wolniewicz, J. Chem. Phys. 103, 1792 (1995). 